\title{
Mapeamento digital de classes de solos: características da abordagem brasileira
}

\author{
Digital soil mapping: characteristics of the brazilian approach
}

\author{
Alexandre ten Caten ${ }^{\mathrm{I}}$ Ricardo Simão Diniz Dalmolin ${ }^{\mathrm{II}}$ Maria de Lourdes Mendonça-Santos ${ }^{\mathrm{III}}$ \\ Elvio Giasson ${ }^{\text {IV }}$
}

\section{- REVISÃO BIBLIOGRÁFICA -}

\section{RESUMO}

\begin{abstract}
O solo é cada vez mais reconhecido como tendo um importante papel nos ecossistemas, assim como para a produção de alimentos e regulação do clima global. Por esse motivo, a demanda por informações relevantes e atualizadas em solos é crescente. Pesquisadores em ciência do solo estão sendo demandados a gerar informações em diferentes resoluções espaciais e com qualidade associada dentro do que está sendo chamado de Mapeamento Digital de Solos (MDS). Devido ao crescente número de trabalhos relacionados ao MDS, faz-se necessário reunir e discutir as principais características dos estudos relacionados ao mapeamento digital de classes de solos no Brasil, o que irá possibilitar uma perspectiva mais ampla dos caminhos, além de nortear trabalhos e demandas futuras. $O$ mapeamento de classes de solos empregando técnicas de MDS é recente no país, com a primeira publicação em 2006. Entre as funções preditivas utilizadas, predomina o emprego da técnica de regressões logísticas. $O$ fator de formação relevo foi empregado na totalidade dos estudos revisados. Quanto à avaliação da qualidade dos modelos preditivos, o emprego da matriz de erros e do índice kappa têm sido os procedimentos mais usuais. A consolidação dessa abordagem automatizada como ferramenta auxiliar ao mapeamento convencional passa pelo treinamento dos jovens pedólogos para a utilização de tecnologias da geoinformação e de ferramentas quantitativas dos aspectos de variabilidade do solo.
\end{abstract}

Palavras-chave: pedometria, classes de solos, levantamento de solos.

\section{ABSTRACT}

Soil is increasingly being recognized as having an important role in ecosystems, as well as for food production and global climate regulation. For this reason, the demand for relevant and updated soil information is increasing. Soil science researchers are being demanded to produce information in different spatial resolutions with associated quality in what is being called Digital Soil Mapping (DSM). Due to an increasing number of papers related to the DSM in Brazil, it is necessary to discuss the main characteristics of those studies related to the automated mapping of soil classes, which will enable a broader perspective of the subject and guide future works and demands. The mapping of soil classes using DSM techniques is recent in the country, the first publication in this topic occurred just in 2006. Among the predictive functions the predominant is logistic regression. The soil formation factor relief was used in all studies reviewed. Quality of predictive models was evaluated employing error matrix and kappa which were the most common procedures. The consolidation of this automated approach as an auxiliary tool to the conventional soil mapping will demand training of young soil scientists to use geoinformation technologies and quantitative tools to handle aspects of soil variability.

Key words: pedometric, soil classes, soil survey.

\section{INTRODUÇÃO}

Há um crescente entendimento da importância do solo para o adequado funcionamento dos ecossistemas, da mesma forma que para a segurança alimentar e ambiental do planeta. Por esse motivo, a demanda por informações relevantes e

'Universidade Federal de Santa Catarina (UFSC), Campus Curitibanos, 89520-000, Curitibanos, SC, Brasil. E-mail: alexandre.ten.caten@ufsc.br. Autor para correspondência.

"Departamento de Solos, Centro de Ciências Rurais (CCR), Universidade Federal de Santa Maria (UFSM), Santa Maria, RS, Brasil. II'Empresa Brasileira de Pesquisa Agropecuária (Embrapa), Centro Nacional de Pesquisa de Solos (CNPS), Rio de Janeiro, RJ, Brasil. ${ }^{\text {Iv } D e p a r t a m e n t o ~ d e ~ S o l o s, ~ F a c u l d a d e ~ d e ~ A g r o n o m i a, ~ U n i v e r s i d a d e ~ F e d e r a l ~ d o ~ R i o ~ G r a n d e ~ d o ~ S u l ~(U F R G S), ~ P o r t o ~ A l e g r e, ~ R S, ~ B r a s i l . ~}$ 
atualizadas em solos está em uma crescente. Contudo, a comunicação da informação acerca do solo é um desafio, devido à divergência de termos, desatualização, generalização e imprecisão dos métodos (SANCHEZ, 2009). A comunidade científica da ciência do solo tem sido um tanto introspectiva e nem sempre fornecendo respostas quantitativas para antigas questões, de modo que essas respostas possam ser utilizadas diretamente pelos usuários do solo ou por tomadores de decisão (HARTEMINK \& MCBRATNEY, 2008).

Entre as oportunidades para a ciência do solo, estão as várias técnicas e métodos já disponíveis aos cientistas de solos, além dos softwares e hardwares que necessitam serem explorados e melhorados. Entre essas oportunidades, está o Mapeamento Digital de Solos (MDS).

O termo Pedometria foi criado pelo pesquisador Alex McBratney da Universidade de Sydney para descrever o estudo quantitativo da variação do solo. A predição e o MDS tiveram suas bases estabelecidas por MCBRATNEY et al. (2003) e definidas por LAGACHERIE \& MCBRATNEY (2007) como "a criação e a população de sistemas de informação espacial de solos por meio de modelos numéricos, visando a inferir as variações espaciais e temporais de classes e propriedades do solo, a partir de observações, conhecimento e dados de covariáveis ambientais relacionados".

Como principal aplicação dessa abordagem, está a predição por meio de modelos matemáticos, das classes e propriedades de solos e o mapeamento digital dos resultados de forma contínua e espacial, criando a possibilidade de organizar um amplo conjunto de dados para análise e interpretações em qualquer época, não sendo o mapa o único produto (MCBRATNEY et al., 2003). Essa abordagem iniciou nos anos 70 e teve grande desenvolvimento nos anos 80, devido aos avanços tecnológicos nas áreas de tecnologia da informação, sensoriamento remoto, estatística, modelagem, posicionamento global, sistemas de medida e, mais recentemente, acesso instantâneo à informação através da rede mundial de computadores.

Considerando o número crescente de trabalhos publicados a respeito da aplicação da técnica de mapeamento digital de classes de solos no Brasil, a presente revisão bibliográfica tem por objetivo reunir e discutir as principais características dos trabalhos já realizados, o que irá possibilitar uma perspectiva mais ampla dos caminhos que o mapeamento automatizado de solos vem seguindo no país, além de nortear trabalhos e demandas futuras.

\section{DESENVOLVIMENTO}

\section{Metodologia adotada}

Artigos relacionados ao mapeamento digital de classes de solos realizados no Brasil foram reunidos neste estudo. Para que o artigo publicado fosse selecionado para essa revisão, ele deveria ter aplicado o MDS stricto sensu. Interpreta-se o sensu stricto como o emprego de covariáveis preditoras, de uma ou mais funções matemáticas e de um conjunto de dados de treinamento. Abordagens lato sensu do MDS, nas quais não ocorre uma classificação numérica de solos, mas apenas uma delimitação de classes de solos apoiada por sistemas informatizados, não foram incluídos neste estudo.

Efetuou-se uma busca nas plataformas de dados Scielo, Scopus e Web of Science, utilizando palavras chaves relacionadas ao tema 'mapeamento digital de classes de solos'. A busca não fez distinção entre revistas científicas nacionais ou estrangeiras.

\section{Temporalidade do MDS no Brasil}

Seguindo os critérios apresentados, onze estudos foram reunidos e as principais informações tabuladas para fomentar a discussão nesta revisão. O mapeamento de classes de solos empregando técnicas de MDS é recente no país (Tabela 1). A primeira publicação nesse sentido ocorreu em 2006, mesmo ano em que foi sediado, no Rio de Janeiro, o $2^{\circ}$ Workshop Global em Mapeamento Digital de Solos. Esse evento pioneiro visou a divulgar entre os cientistas do solo brasileiros os métodos e técnicas empregados no MDS.

Esse despertar mais recente dos cientistas do solo brasileiros para o MDS pode estar ligado (i) à disponibilização bem mais tardia de software e hardware no país; (ii) ao conservadorismo de muitos pedólogos que relutam em utilizar sistemas automatizados capazes de contribuir para o mapeamento de solos; (iii) à carência de pessoal qualificado para o emprego da tecnologia da informação na ciência do solo; (iv) à popularização mais recente no Brasil de tecnologias como sistema de posicionamento global e sensoriamento remoto. Em comparação, o mapeamento de classes de solos empregando técnicas automatizadas já vem sendo praticado há muitos anos em outros países. WEBSTER \& BURROUGH(1972) já demonstraram, há quase quatro décadas, o emprego de estatística multivariada aplicada em sistemas informatizados para mapear classes de solos no sul da Inglaterra.

Estudos relacionados ao MDS estão se tornando mais frequentes. Em 2011, foram cinco trabalhos publicados (CARVALHO JUNIOR et al., 2011; GIASSON et al., 2011; TEN CATEN et al., 2011a; TEN 
Tabela 1 - Informações descritivas de onze estudos em mapeamento digital de classes de solos realizados no Brasil.

\begin{tabular}{|c|c|c|c|c|c|c|}
\hline Estudos & Modelos & Fatores & Atributos usados & Locais & Escalas & Área $\left(\mathrm{km}^{2}\right)$ \\
\hline CARVALHO JUNIOR et al. (2011) & NEU & $\mathrm{r}, \mathrm{s}$ & $\begin{array}{l}\text { ele, dec, pla, per, cur, } \\
\text { asp, acu, dir, elr, deh }\end{array}$ & $\begin{array}{l}\text { Extremo norte do Estado } \\
\text { do Rio de Janeiro. }\end{array}$ & $1: 250.000$ & 164,7 \\
\hline GIASSON et al.(2011) & $\mathrm{ADE}$ & r, s & $\begin{array}{l}\text { dec, pla, per, umi, cur, } \\
\text { dir, acu, com, pod }\end{array}$ & Bento Gonçalves (RS) & $1: 10.000$ & 6,14 \\
\hline TEN CATEN et al. (2011a) & REG & $\mathrm{r}, \mathrm{c}, \mathrm{s}$ & $\begin{array}{l}\text { ele, dec, pla, per, dis, } \\
\text { con, umi, sed, rad }\end{array}$ & São Pedro do Sul (RS) & $1: 50.000$ & 874 \\
\hline TEN CATEN et al. (2011b) & REG & $\mathrm{r}, \mathrm{c}, \mathrm{s}$ & $\begin{array}{l}\text { ele, dec, pla, per, dis, } \\
\text { con, umi, sed, rad }\end{array}$ & São Pedro do Sul (RS) & $1: 50.000$ & 874 \\
\hline TEN CATEN et al. (2011c) & REG & $\mathrm{r}, \mathrm{c}, \mathrm{s}$ & $\begin{array}{l}\text { ele, dec, pla, per, dis, } \\
\text { con, umi, sed, rad }\end{array}$ & São Pedro do Sul (RS) & $1: 50.000$ & 874 \\
\hline CHAGAS et al.(2010) & NEU & $\begin{array}{l}\mathrm{r}, \mathrm{p}, \\
\mathrm{o}, \mathrm{s}\end{array}$ & $\begin{array}{l}\text { ele, dec, pla, umi, asp, } \\
\text { geo, arg, oxi, ndv }\end{array}$ & $\begin{array}{l}\text { Bacia hidrográfica do Rio } \\
\text { São Domingos (RJ) }\end{array}$ & $\begin{array}{l}1: 100.000 \\
\text { a } 1: 50.000\end{array}$ & 9,9 \\
\hline COELHO \& GIASSON (2010) & $\begin{array}{l}\text { REG, } \\
\text { ADE, } \\
\text { BAY, } \\
\text { CAR, } \\
\text { LMT }\end{array}$ & r, s & $\begin{array}{l}\text { dec, pla, per, umi, cur, } \\
\text { dir, acu }\end{array}$ & $\begin{array}{l}\text { Ijuí, Bozano e Coronel } \\
\text { Barros (RS) }\end{array}$ & 1:50.000 & 1.018 \\
\hline CRIVELENTI et al. (2009) & $\mathrm{ADE}$ & $\mathrm{r}, \mathrm{p}$ & $\begin{array}{l}\text { dec, pla, per, dis, con, } \\
\text { geo }\end{array}$ & $\begin{array}{l}\text { Carta topográfica Dois } \\
\text { Córregos (SP) }\end{array}$ & $1: 100.000$ & 772 \\
\hline CARVALHO et al.(2009) & NEB & $\mathrm{r}, \mathrm{p}, \mathrm{o}$ & ele, dec, geo, veg & Mucugê (Ba) & $1: 50.000$ & 382,2 \\
\hline FIGUEIREDO \& GIASSON (2008) & REG & $\mathrm{r}, \mathrm{s}$ & $\begin{array}{l}\text { dec, pla, per, dis, umi, } \\
\text { dir, acu, com }\end{array}$ & $\begin{array}{l}\text { Ibirubá e Quinze de } \\
\text { Novembro (RS) }\end{array}$ & $1: 80.000$ & 720 \\
\hline GIASSON et al. (2006) & REG & r, s & $\begin{array}{l}\text { dec, pla, per, umi, cur, } \\
\text { dir, acu, com, pod }\end{array}$ & Sentinela do Sul (RS) & 1:50.000 & 253 \\
\hline
\end{tabular}

REG: regressões logísticas múltiplas, ADE: árvore de decisão, BAY: classificação Bayes, CAR: classificação hierárquica CART, LMT: classificação hierárquica LMT, NEU: redes neurais, NEB: lógica nebulosa, r: relevo, c: clima, s: solo, p: material de origem, o: organismos, ele: elevação, dec: declividade, pla: curvatura planar, per: curvatura de perfil, dis: distância à drenagem, con: área de contribuição, umi: índice de umidade topográfica, sed: capacidade de transporte de sedimento, cur: curvaturas combinadas, dir: direção de fluxo, acu: fluxo acumulado, com: comprimento de fluxo, pod: índice de poder de córrego, asp: aspecto, geo: geologia, arg: índice de argila, oxi: óxido de ferro, ndv: NDVI, veg: vegetação, rad: radiação relativa disponível, elr: elevação relativa, deh: distância euclidiana à hidrografia.

CATEN et al., 2011b; TEN CATEN et al., 2011c). Com a criação da Comissão Especializada em Pedometria dentro da divisão Solo no Espaço e no Tempo pela Sociedade Brasileira de Ciência do Solo (SBCS), haverá uma maior divulgação do MDS entre cientistas do solo no Brasil.

Modelos preditivos empregados

Entre as funções matemáticas utilizadas, predomina o emprego da técnica de regressões logísticas (Tabela 1). Através de uma relação linearizada entre covariáveis preditoras e classes de solos, as regressões logísticas geram um valor de pertinência para cada classe de solos a ser mapeada sobre a paisagem (GIASSON et al., 2006). Esses valores de probabilidade para um mesmo ponto da paisagem são comparados e a classe de solo com maior valor é atribuída a posição em análise, gerando-se um mapa de classe de solos. As regressões logísticas apresentam como desvantagem uma sensibilidade à proporção relativa entre classes de solos no conjunto de treinamento dos modelos e uma relativa complexidade para a interpretação dos parâmetros estatísticos dos modelos logísticos gerados (TEN 
CATEN et al., 2011). Por outro lado, as regressões logísticas têm como vantagem o fato de lidar com covariáveis preditoras contínuas, discretas ou binárias, além de gerar classes de solos com valor de probabilidade de ocorrência associado.

Os estudos que empregam a técnica de mineração de dados por Árvore de Decisão (AD) têm como objetivo utilizar uma abordagem quantitativa a qual relaciona os fatores de formação e classes de solos de forma similar ao raciocínio do pedólogo (CRIVELENTI et al., 2009; GIASSON et al., 2011). O emprego de AD é fruto de pesquisas que buscam aplicar técnicas robustas para a extração de padrões em grandes conjuntos de dados (WITTEN \& FRANK, 2005). A abordagem por AD tem sido empregada por apresentar a vantagem de possibilitar a expressão das relações solo-paisagem de maneira explícita, o agrupamento e a busca por padrões, além de possibilitar o entendimento de como esses dados são interrelacionados (KHEIR et al., 2010). Todavia, nos estudos até aqui realizados no país, não se tem dado ênfase à análise das regras de decisão geradas durante a modelagem, o que permite afirmar que a $\mathrm{AD}$ vem sendo empregada principalmente pela sua robustez como técnica preditiva, com mais ênfase ao mapa final gerado do que ao seu potencial em explicitar e esclarecer as relações entre fatores de formação e classes de solos.

Entre as estratégias referidas como 'caixa preta' (BALLABIO, 2009), a técnica de redes neurais artificiais (RNA) foi empregada por CHAGAS et al. (2009) e CARVALHO JUNIOR et al. (2011). O uso de redes neurais possibilita uma grande acurácia na predição, contudo, os problemas aparecem quando a rede precisa ser aplicada como conhecimento formalizado, devido à complexidade da rede e aos pesos empregados (QI \& ZHU, 2003). KHEIR et al. (2010) concordam com essa dificuldade para a implementação das RNA, afirmando que as redes neurais não possibilitam um modelo de fácil entendimento que permita aos pesquisadores terem um entendimento completo da natureza dos dados em análise.

\section{Fatores de formação empregados}

O fator solo foi usado na maioria dos estudos revisados, dado que os modelos são calibrados utilizandose áreas de referência com mapeamento de solo, em que a distribuição dos solos é a variável dependente. $O$ fator de formação relevo foi empregado na totalidade dos estudos (Tabela 1) devido a sua ampla disponibilidade através de Modelo Digital de Elevação (MDE), derivado do SRTM (Shuttle Radar Topography Mission) e de cartas topográficas. Também contribui para o emprego do relevo a possibilidade de derivar a partir dele um maior número de covariáveis preditoras e a clara relação existente entre o relevo e o padrão de distribuição espacial das classes de solos. O MDE é uma das principais fontes de informação para o MDS e modelos de elevação derivados de cartas topográficas e do instrumento ASTER, no satélite Terra, necessitam ser avaliados.

Os fatores material de origem e clima foram utilizados com menor frequência (Tabela 1). Isso decorre possivelmente da generalização dos mapas disponíveis dessas informações. Os mapas geológicos amplamente disponíveis para todo o território nacional encontramse na escala 1:1.000.000. Já a informação acerca do clima, além da questão da falta de resolução nos mapas disponíveis, existe ainda o fato de que os solos tenham se formado em tempos pretéritos, podendo haver uma baixa correlação entre condições climáticas atuais e as classes de solos. Além disso, o clima é um fator que apresenta um efeito pedogenético mais expressivo em escala regional, enquanto a maioria dos trabalhos envolvendo MDS apresentam escalas maiores, em que, muitas vezes, não há registro de variação climática.

\section{Distribuição e dimensão dos estudos}

Entre os onze estudos analisados, sete foram realizados no Estado do Rio Grande do Sul (Tabela 1). Isso pode ser atribuído à presença de dois grupos de pesquisa, os quais desenvolvem atividades nessa linha naquele Estado. É importante que essa técnica seja divulgada e avaliada em outras regiões do país. Essa informação contrasta com os vazios de levantamentos de solos reportados por MENDONÇA-SANTOS \& SANTOS (2007). Esses autores descrevem que as regiões Norte e Noroeste, sobretudo grandes áreas de floresta Amazônica, possuem informação apenas em pequena escala sobre os solos da região.

Como a aplicação do MDS implica uma fase de treinamento dos modelos preditivos, o efetivo auxílio dessa técnica para o aumento do conhecimento a respeito da distribuição espacial dos solos nas regiões norte e noroeste só irá ocorrer a partir da disponibilização dessas informações.

O MDS aplicado à predição de classes de solos no Brasil tem ocorrido predominantemente em nível semidetalhado (1:50.000) (Tabela 1), possivelmente devido à disponibilidade de mapas de solos para serem usados como áreas de referência ou ao emprego de cartas topográficas nesta escala para a geração de MDE.

Mapas de solos, disponíveis para o limite de alguns municípios, têm sido o conjunto de dados mais utilizado para treinamento dos modelos preditivos (Tabela 1). Estudos em que foram aplicados os limites administrativos dos municípios podem ter sua posterior utilização dificultada pelo fato de uma bacia hidrográfica 
abranger vários municípios. Esse mesmo problema ocorre caso sejam utilizados dados oriundos de cartas topográficas. Os mapas de solos utilizados têm condicionado a extensão territorial da maioria dos trabalhos.

Procedimentos metodológicos

A densidade de amostragem utilizada para a calibração dos modelos é muito ampla, variando de 10 amostras $/ \mathrm{km}^{2}$ a 1.111 amostras $/ \mathrm{km}^{2}$, indicando que existe uma carência pela padronização do número de amostras a ser utilizada na fase de treinamento dos modelos (Tabela 2).

A totalidade dos estudos utilizou de MDE, como já mencionado, possivelmente devido a questões ligadas ao fator de formação relevo. Entre as fontes de MDE utilizadas, destaca-se o SRTM (Shuttle Radar Topography Mission). Esse dado tem sido utilizado em sua resolução original de $90 \mathrm{~m}$, ou no formato Topodata (VALERIANO \& ROSSETTI, 2010), que, por sua vez, consiste em uma interpolação dos dados SRTM para 30m. As curvas de nível nas cartas topográficas também têm sido utilizadas como fontes de dados altimétricos. A utilização de tecnologias como o LIDAR, satélite TerraSAR-X, Scanners 3D e GNSS RTK para a obtenção da informação sobre as nuances do terreno ainda não foram testadas no MDS nacional. Essas tecnologias têm um grande potencial na obtenção de MDE de elevada resolução e exatidão, visando ao levantamento preditivo de solos em pequenas áreas e/ ou grandes escalas.

Entre os estudos de MDS realizados no país, predomina a utilização de softwares proprietários. Os programas de código fonte fechados podem limitar a comunicação dos procedimentos executados para a obtenção de determinado resultado (WOOD, 2009). Ainda não estão sendo utilizados os programas com código fonte aberto como SAGA, GRASS, ILWIS e o pacote estatístico $\mathrm{R}$, que tem uma grande rede de colaboradores.

Qualidade e disponibilidade da informação

A matriz de erros e do índice kappa têm sido as formas mais empregadas para a avaliação da qualidade dos modelos preditivos (Tabela 3). A matriz

Tabela 2 - Aspectos metodológicos do mapeamento digital de classes de solo no Brasil.

\begin{tabular}{|c|c|c|c|c|c|c|}
\hline Estudos & $\begin{array}{l}\text { Total de } \\
\text { amostras }\end{array}$ & $\begin{array}{l}\text { Amostras } \\
/ \mathrm{km}^{2}\end{array}$ & $\begin{array}{c}\text { Total amostrado } \\
\text { (\%) }\end{array}$ & $\begin{array}{l}\text { Fonte do } \\
\text { MDE }\end{array}$ & $\begin{array}{l}\text { Resolução } \\
\text { (m) }\end{array}$ & Software \\
\hline CARVALHO JUNIOR et al. (2011) & 125 a $359 *$ & $*$ & * & cart & 20 & $\begin{array}{l}\text { arcv, arcg, erda, } \\
\text { exce, java, exec }\end{array}$ \\
\hline GIASSON et al. (2011) & 1.333 & 217 & 0,54 & foto & 5 & arcv, weka \\
\hline TEN CATEN et al. (2011a) & 70.000 & 80 & 20,00 & srtm & 50 & arcg \\
\hline TEN CATEN et al. (2011b) & 58.440 & 67 & 16,72 & srtm & 50 & $\operatorname{arcg}$ \\
\hline TEN CATEN et al. (2011c) & 70.000 & 80 & 20,00 & srtm & 50 & arcg \\
\hline CHAGAS et al.(2010) & 3.000 & 303 & 27,27 & cart & 30 & arcg, erda, java \\
\hline COELHO \& GIASSON (2010) & 11.000 & 11 & 8,75 & srtm & 90 & arcg, weka \\
\hline CRIVELENTI et al. (2009) & 794.273 & 1.029 & 90,00 & cart & 30 & arcg, ilwi, idri, weka \\
\hline CARVALHO et al.(2009) & 424.643 & 1.111 & 100,00 & srtm & 30 & $\operatorname{arcg}$ \\
\hline FIGUEIREDO et al. (2008) & 7.200 & 10 & 8,10 & srtm & 90 & arcv \\
\hline GIASSON et al. (2006) & 7.500 & 30 & 25,00 & srtm & 92 & arcv \\
\hline
\end{tabular}

foto: pares de fotografias estéreo, cart: cartas topográficas, srtm: shuttle radar topographic mission, arcg: ArcGIS 9, arcv: ArcView 3.2, ilwi: Ilwis, idri: Idrisi Andes, erda: Erdas, java: Java Neural Network Simulator, weka: Weka, exce: Excel, exec: Executables funcpow, gerapat e Max_like_cof (Carvalho Junior et al., 2011), *: variáveis conforme a unidade de mapeamento.

Ciência Rural, v.42, n.11, nov, 2012. 
de erros é uma boa alternativa para determinar a natureza e a frequência dos erros envolvidos, contudo, caso a matriz seja gerada de maneira inapropriada, sem ser representativa dos dados, a sua interpretação não terá valor (CONGALTON, 1991).

Entre os estudos, em apenas três casos, foi avaliada a acurácia do mapeamento com dados de campo (CHAGAS et al., 2010; TEN CATEN et al., 2011b; CARVALHO JUNIOR et al., 2011). Predomina entre os estudos apenas a avaliação do potencial de reprodução do mapa original a partir dos modelos preditivos, que pode não ser uma abordagem eficaz para indicar a qualidade do mapa de solos gerado, dado que o mapa original pode apresentar erros.

O valor médio do índice kappa entre os estudos realizados no país é de 0,48 (Tabela 3). Esse valor é similar aos valores reportados na literatura internacional como em HENGL \& ROSSITER (2003), de 0,58 em locais montanhosos e de 0,39 para áreas planas, e por SCULL et al. (2005), que observaram valores de 0,44 e 0,52 em áreas montanhosas e planas, respectivamente. A abordagem adotada em ambos os estudos, pela estratificação da área a ser mapeada de acordo com características locais predominantes, tem sido apresentada como uma possibilidade de melhorar o desempenho dos modelos preditivos, pois possibilita a geração de modelos mais regionalizados, com melhor desempenho em cada uma das regiões para as quais foram desenvolvidos. A abordagem estratificada foi testada por GIASSON et al. (2006), com um valor de kappa de 0,31 para a abordagem estratificada e de 0,36 quando não estratificada. Esses autores relataram que a possível causa do pior desempenho da abordagem estratificada tenha sido o modelo digital de elevação de $90 \mathrm{~m}$ utilizado no estudo.

A predição das classes de solos foi realizada nos quatro níveis categóricos do Sistema Brasileiro de Classificação de Solos (SiBCS). A quase totalidade dos estudos também buscou mapear classes de solos na forma de associações. Alguns estudos buscaram avaliar o potencial das metodologias utilizadas em mapear níveis categóricos mais baixos, como o $3^{\circ}$ e o $4^{\circ}$ níveis do SiBCS. No entanto, nesses níveis, os solos são classificados de acordo com características de mais difícil associação com os fatores de formação do solo, a exemplo de características físicas e químicas no $3^{\circ}$ nível, e da presença de variações e características extraordinárias para a classificação no $4^{\circ}$ nível. Acertos na distinção e na espacialização dessas classes na paisagem podem estar mais sendo fruto do acaso do que do verdadeiro poder preditivo dos modelos.

Alguns estudos têm se utilizado da estratégia de simplificar a legenda, em relação a um mapa de referência, visando a melhorar a capacidade preditiva dos modelos. Esta abordagem consiste em mapear classes na forma de associações de solos, ou no mapeamento de classes apenas em níveis mais elevados do SiBCS. Esta estratégia melhora os indicadores de acerto em relação a um mapa de referência, contudo, produz mapas generalizados com um grau cada vez maior de variações dentro da unidade de mapeamento.

Os resultados das pesquisas em MDS realizadas no país são, na sua maioria, publicados em

Tabela 3 - Características dos estudos revisados quanto a aspectos de qualidade e disponibilidade da informação gerada.

\begin{tabular}{|c|c|c|c|c|c|}
\hline Estudos & Qualidade & Índice kappa* & NC & Legenda simplificada & Idioma \\
\hline CARVALHO JUNIOR et al. (2011) & mat / kap / sim & 0,55 & $1^{\circ}$ & não realizada & Ing \\
\hline GIASSON et al. (2011) & mat / kap / não & 0,52 & $2^{\circ}$ & realizada & Ing \\
\hline TEN CATEN et al. (2011a) & mat / kap / não & $0,63^{* *}$ & ass, $1^{\circ}, 2^{\circ}$ & não realizada & Port \\
\hline TEN CATENcaten et al. (2011b) & mat / kap / sim & 0,46 & ass, $2^{\circ}$ & não realizada & Port \\
\hline TEN CATEN et al. (2011c) & mat / kap / não & 0,37 & ass, $2^{\circ}$ & não realizada & Port \\
\hline CHAGAS et al.(2010) & mat / kap / sim & 0,73 & ass, $4^{\circ}$ & realizada & Port \\
\hline COELHO \& GIASSON (2010) & mat / kap / não & 0,38 & $2^{\circ}, 3^{\circ}, 4^{\circ}$ & realizada & Port \\
\hline CRIVELENTI et al. (2009) & mat / kap / não & 0,43 & ass, $3^{\circ}, 4^{\circ}$, textura & realizada & Port \\
\hline CARVALHO et al.(2009) & visual / não & - & ass, $2^{\circ}, 3^{\circ}, 4^{\circ}$ & não realizada & Port \\
\hline FIGUEIREDO \& GIASSON (2008) & mat / kap / não & $0,38 * * *$ & ass, $3^{\circ}$, relevo, textura & realizada & Port \\
\hline GIASSON et al. (2006) & mat / kap / não & $0,36 * * *$ & ass, $1^{\circ}, 2^{\circ}$ & realizada & Ing \\
\hline
\end{tabular}

NC: nível categórico do SiBCS, mat: matriz de erros, kap: índice kappa, visual: comparação visual entre mapas, / sim: verificado no campo, / não: não verificado no campo, ass: associações, AMM: área mínima mapeavel, *: reprodutibilidade do mapa, **: dado não publicado, ***: sem simplificação da legenda, Ing: Inglês, Port: Português. 
artigos em língua portuguesa (Tabela 3). Como o Brasil é um país com uma diversidade de biomas e com grandes lacunas na cartografia de seus solos, os resultados aqui alcançados, sem dúvida, são de interesse da comunidade internacional. Entre as experiências já relatadas do MDS, predominam as condições de solos temperados e de regiões áridas do globo (MCBRATNEY et al., 2003; SCULL et al., 2003; GRUNWALD, 2009). Faz-se fundamental que os estudos dessa linha de pesquisa, realizados em condições de clima tropical, sejam divulgados internacionalmente.

\section{Expectativas para o MDS no Brasil}

Os custos do MDS não foram mencionados em nenhum dos estudos revisados. Para GRUNWALD (2009), a pesquisa relacionada aos investimentos necessários para tornar o MDS efetivo na produção de conhecimento espacial acerca de classes e propriedades de solos necessita mais atenção dos pesquisadores. Os custos envolvidos no acesso aos levantamentos de solos já existentes e a coleta de novas informações, assim como os dados oriundos de técnicas de sensoriamento proximal ou remoto deverão ser considerados. O conhecimento de especialistas em solos, estatística, informática e geomática precisam ser avaliados no custo total de projetos em MDS. O tempo investido em um projeto de MDS também deverá ser relatado em futuros estudos, para que novos projetos possam ser adequadamente planejados.

Uma padronização da abordagem para a avaliação da qualidade dos mapas gerados será fundamental. À medida que os dados existentes (mapas de solos) conjuntamente com os dados de apoio de fácil aquisição (SRTM), os quais possuem erros inerentes, são cada vez mais utilizados para o MDS, é preciso que uma metodologia padronizada seja proposta para avaliar e possibilitar comparações entre resultados. Espera-se dos sistemas modernos para o mapeamento de solos mais qualidade do que dos mapeamentos convencionais, pois, no MDS, são empregados dados melhor definidos e documentados em termos de posicionamento, atualização e integração de dados (FINKE, 2007). A avaliação da qualidade dos mapas de classes de solos a partir dos resultados da matriz de erros e índice kappa em relação ao mapa original de solos pode estar subestimando a verdadeira qualidade dos mapas gerados pelo MDS. Como os mapas convencionais são a representação cartográfica do conhecimento pedológico empregado na sua produção e o conhecimento tácito do pedólogo está em constante aprimoramento, haverá sempre distorções entre o mapa gerado pelo pedólogo e a verdadeira classe de solo presente na paisagem. De tal forma que a comparação do mapa gerado pela metodologia automatizada com aquele obtido pela metodologia convencional apenas indica a capacidade de reprodução deste último pelo modelo preditivo. A checagem do mapa predito com informações de campo deve ser o verdadeiro indicador de qualidade a ser empregado.

Como uma das prerrogativas para a execução de um projeto de MDS é a sua implementação em um sistema de informação geográfica, a disponibilização da informação gerada em formatos vetorial ou matricial é facilitada. Contudo, não é o que tem sido relatado nos estudos realizados no país. Em nenhum dos artigos revisados, os autores relatam a disponibilização dos mapas gerados.

\section{CONSIDERAÇÕES FINAIS}

Os pedólogos têm se questionado sobre as motivações para a redução da valorização de sua atividade profissional. Entre os culpados são elencadas a valorização da pesquisa aplicada em detrimento à básica, os critérios taxonômicos utilizados pela pedologia, os planejadores sem interesse pela atividade do pedólogo, levantamentos pedológicos insuficientes para aplicação imediata em problemas da sociedade (KER \& NOVAIS, 2003). Em contraste com a argumentada recessão verificada em relação aos levantamentos de solos, verifica-se uma tendência de renovação, principalmente, quanto às técnicas de coleta e processamento de dados, formação e desenvolvimento de modelos e ao MDS (RAMOS, 2003).

Há a necessidade de que sejam somados esforços para o treinamento de novos pedólogos aptos a implementar projetos de MDS, bem como em realizar levantamentos de solos por meio das metodologias convencionais. Ambas as abordagens são complementares e necessitam ser desenvolvidas simultaneamente. O levantamento de solos convencional produz as informações que são utilizadas para treinar os modelos e predizer classes e propriedades de solos em áreas não mapeadas. A abordagem automatizada permite que extensas regiões sejam previamente mapeadas, otimizando recursos humanos e financeiros no mapeamento convencional.

\section{REFERÊNCIAS}

BALLABIO, C. Spatial prediction of soil properties in temperate mountain regions using support vector regression, Geoderma, v.151, n.3-4, p.338-350, 2009. Disponível em: <http:// www.sciencedirect.com/science/article/pii/S0016706109001499>. Acesso em: 12 out. 2011. doi: 10.1016/j.geoderma.2009.04.022.

CARVALHO, C.C.N. Mapa digital de solos: Uma proposta metodológica usando inferência fuzzy. Revista Brasileira de Engenharia Agrícola 
e Ambiental, v.13, n.1, p.46-55, 2009. Disponível em: <http:// www.scielo.br/scielo.php?script=sci_arttext\&pid=S1415-436620090 00100007\&lng=en\&nrm=iso>. Acesso em: 25 mai. 2011. doi: 10.1590/ S1415-43662009000100007.

CARVALHO JUNIOR, W. de. et al. Digital soilscape mapping of tropical hillslope areas by neural networks. Scientia Agricola, v.68, n.6, p.691-696, 2011. Disponível em: <http://www.scielo.br/ scielo.php? script =sci_art text \& pid = S 0103 90162011000600014\&lng=en \&nrm=iso>. Acesso em: 20 jan. 2012. doi: http://dx.doi.org/10.1590/S0103-90162011000600014.

CHAGAS, C. da S. Atributos topográficos e dados do Landsat7 no mapeamento digital de solos com uso de redes neurais. Pesquisa Agropecuária Brasileira, v.45, n.5, p.497-507, 2010. Disponível em: <http://www.scielo.br/scielo.php?script=sci_arttext\&pid=S141543662009000100007\&lng=en\&nrm=iso>. Acesso em: 25 mai. 2011. doi: $10.1590 /$ S1415-43662009000100007.

COELHO, F.F.; GIASSON, E. Comparação de métodos para mapeamento digital de solos com utilização de sistema de informação geográfica. Ciência Rural, v.40, n.10, p.2099-2106, 2010. Disponível em: <http://www.scielo.br/scielo.php?script=sci_arttext\&pid=S010384782010001000008\&lng=en\&nrm=iso>. Acesso em: 20 fev. 2011. doi: 10.1590/S0103-84782010005000156.

CONGALTON, R.G. A Review of Assessing the Accuracy of Classifications of Remotely Sensed Data. Remote Sensing of Environment, v.37, p.35-46, 1991. Disponível em: <http:// www.sciencedirect.com/science/article/pii/003442579190048B >. Acesso em: 12 out. 2011. doi: 10.1016/0034-4257(91)90048-B.

CRIVELENTI, R.C. et al. Mineração de dados para a inferência de relações solo-paisagem em mapeamentos digitais de solo. Revista Agropecuária Brasileria, v.44, n.12, p.1707-1715, 2009. Disponível em: <http://www.scielo.br/scielo.php?script=sci_arttext\&pid=S0100204X2009001200021\&lng=en\&nrm=iso>. Acesso em: 25 mai. 2011. doi: 10.1590/S0100-204X2009001200021.

FIGUEIREDO, S.R. et al. Uso de regressões logísticas múltiplas para mapeamento digital de solos no planalto médio do RS. Revista Brasileira de Ciência do Solo, v.32, p.2779-2785, 2008. Disponível em: <http://www.scielo.br/scielo.php?script=sci_arttext\&pid=S010006832008000700023\&lng=en\&nrm=iso>. Acesso em: 20 fev. 2011. doi: 10.1590/S0100-06832008000700023.

GIASSON, E. et al. Decision trees for digital soil mapping on subtropical basaltic steeplands. Scientia Agrícola, v.68, p.167174, 2011. Disponível em: <http://www.scielo.br/ scielo.ph p ? script =sci_art text \& pid = S 0103 90162011000200006\&lng $=$ en $\&$ nrm=iso>. Acesso em: 25 mai. 2011. doi: 10.1590/S0103-90162011000200006.

GIASSON, E. et al. Digital soil mapping using multiple logistic regression on terrain parameters in southern Brazil. Scientia Agricola, v.63, p.262-268, 2006. Disponível em: <http:// www.scielo.br/scielo.php?script=sciarttext\&pid=S0103$90162006000300008 \& \operatorname{lng}=$ en $\& \mathrm{nrm}=\mathrm{iso}>$. Acesso em: 20 fev. 2011. doi: 10.1590/S0103-90162006000300008.

GRUNWALD, S. Multi-criteria characterization of recent digital soil mapping and modeling approaches. Geoderma, v.152, p.195207. 2009. Disponível em: <http://www.sciencedirect.com/science/ article/pii/S0016706109001827>. Acesso em: 12 out. 2011. doi: 10.1016/j.geoderma.2009.06.003.
HARTEMINK, A.E.; MCBRATNEY, A.B., A soil science renaissance. Geoderma, v.148, n.2, p.123-129, 2008. Disponível em: <http://www.sciencedirect.com/science/article/ pii/S0016706108002802>. Acesso em: 12 out. 2011. doi: 10.1016/j.geoderma.2008.10.006.

HENGL, T.; ROSSITER, D.G. Supervised Landform classification to enhance and replace photo-interpretation in semi-detailed soil survey. Soil Science Society of America Journal, v.67, n.6, p.1810-1822, 2003. Disponível em: <https://www.soils.org/publications/sssaj/ abstracts/67/6/1810>. Acesso em: 20 fev. 2012. doi: 10.2136/sssaj2003.1810.

KER, J.C.; NOVAIS, R.F. Fundamentos da pedologia e relação com a fertilidade do solo, 2003, Ribeirão Preto, SP. In: XXIX CONGRESSO BRASILEIRO DE CIÊNCIA DO SOLO, 29, 2003, Ribeirão Preto, SP. Anais... Viçosa: Sociedade Brasileira de Ciência do Solo,- 2003. 27p.

KHEIR, R.B. et al. Spatial soil zinc content distribution from terrain parameters: A GIS-based decision-tree model in Lebanon. Environmental Pollution, v.158, n.2, p.520-528, 2010. Disponível em: <http://www.sciencedirect.com/science/article/ pii/S0269749109004163>. Acesso em: 12 out. 2011. doi: 10.1016/j.envpol.2009.08.009.

LAGACHERIE, P.; MCBRATNEY, A. B. Spatial soil information systems and spatial soil inference systems: perspectives for digital soil mapping. In: LAGACHERIE, P. et al. Digital soil mapping: an introductory perspective. Amsterdam: Elsevier, 2007, Cap. 1, p. 3-22.

MCBRATNEY, A. B. et al. On digital soil mapping. Geoderma, v.17, p.3-52, 2003. Disponível em: <http://www.sciencedirect.com/ science/article/pii/S0016706103002234>. Acesso em: 12 0ut. 2011. doi: 10.1016/S0016-7061(03)00223-4.

MENDONÇA-SANTOS, M.L.; SANTOS, H.G. dos The state of the art of brazilian soil mapping and prospects for digital soil mapping. In: LAGACHERIE, P. et al. Digital soil mapping: an introductory perspective. Amsterdam: Elsevier, 2007, Cap.3, p.39-54.

QI, F.; ZHU, A. X. Knowledge discovery from soil maps using inductive learning. International Journal of Geographical Information Science. v.17, n.8, p.771-795, 2003. Disponível em: <http:// solimserver.geography.wisc.edu/pdfs/QiFeng_IJGIS2003.pdf>. Acesso em: 12 out. 2011. doi: 10.1080/13658810310001596049.

RAMOS D.P. Desafios da Pedologia Brasileira frente ao novo milênio - (CNPS/EMBRAPA). In: Simpósio do XXIX Congresso Brasileiro de Ciência do Solo, Ribeirão Preto, SP, 2003. Anais... Viçosa: Sociedade Brasileira de Ciência do Solo,2003. 9p.

SANCHEZ, P.A. et al. Digital soil map of the world. Science, v.325, p.680-681, 2009. Disponível em: <http://www.sciencemag.org/ content/325/5941/680.full.pdf.> Acesso em: 12 out. 2011. doi: 10.1126/science. 1175084 .

SCULL, P. et al. Predictive soil mapping: a review. Progress in Physical Geography, v.27, p.171-197, 2003. Disponível em: <http://ppg.sagepub.com/content/27/2/171.full.pdf>. Acesso em: 06 jan. 2012. doi: 10.1191/0309133303pp366ra.

TEN CATEN, A. et al. Regressões logísticas múltiplas: fatores que influenciam sua aplicação na predição de classes de solos. Revista 
Brasileira de Ciência do Solo, v.35, n.1, p.53-62, 2011a. Disponível em: <http://www.scielo.br/scielo.php?script=sci_arttext\&pid=S0100$06832011000100005 \& \operatorname{lng}=$ en\&nrm=iso>. Acesso em: 16 mai. 2011. doi: 10.1590/S0100-06832011000100005.

TEN CATEN, A. et al. Extrapolação das relações solo-paisagem a partir de uma área de referência. Ciência Rural, v.41, n.5, p. 812-816, 2011b. . Disponível em: <http://www.scielo.br/ s c i e l o.ph p ? s c ri p t = s ci_art t ext \& pid = S 0103 84782011000500012\&lng $=$ en\&nrm=iso $>$. Acesso em: 12 out. 2011. doi: 10.1590/S0103-84782011000500012.

TEN CATEN, A. et al. Componentes principais como preditores no mapeamento digital de classes de solos. Ciência Rural, v.41, n.7, p. 1170-1176, 2011c. Disponível em: <http://www.scielo.br/ s c i elo.php ? s cript = s ci__art text \& pid = S 0103 84782011000700011\&lng $=$ en\&nrm=iso $>$. Acesso em: 12 out. 2011. doi: 10.1590/S0103-84782011000700011.

VALERIANO, M. M.; ROSSETTI, D. F. Topodata: seleção de coeficientes geoestatísticos para refinamento unificado de dados
SRTM. São José dos Campos, SP: NPE: Coordenação de Ensino, Documentação e Programas Especiais (INPE-16701-RPQ/853) (Boletim). 2010. 74p.

WEBSTER, R.; BURROUGH, P.A. Computer-based soil mapping of small areas from sample data. 1. Multivariate classification and ordination. Journal of Soil Science, v.23, p.210-221, 1972. Disponível em: <http://dx.doi.org/10.1111/ j.1365-2389.1972.tb01654.x>. Acesso em: 12 out. 2011. doi.: 10.1111/j.1365-2389.1972.tb01654.x.

WITTEN, I.H.; FRANK, E. Data Mining: practical machine learning tools and techniques, $2^{\text {nd }}$ ed., Burlington: Morgan Kaufmann, 2005, 560p.

WOOD, J. Overview of software packages used in geomorphometry. In: HENGL, T.; REUTER, H.I. Geomorphometry: Concepts, Software, Applications. Amsterdam: Elsevier, 2009, Cap.10. p.257-267. 